\title{
Cuando un príncipe besa a una rana: ¡Sorpresa! Una propuesta didáctica desde la homosexualidad
}

JOSÉ ANTONIO JÓDAR SÁNCHEZ

Universitat de Barcelona / University at Buffalo, State University of New York

ROCÍO DOMENE BENITO

Universitat de València

\begin{abstract}
Resumen: En esta propuesta didáctica, utilizamos el álbum ilustrado como herramienta para la enseñanza de contenido lingüístico, así como valores sociales y cívicos a un grupo de estudiantes de ELE de nivel A2. Utilizamos el álbum ilustrado El día de la rana roja que narra la historia de dos príncipes que se enamoran y adoptan a una niña huérfana. Estructuramos nuestra propuesta en cuatro fases (introducción, comprensión, consolidación, resignificación), de las cuáles las dos últimas son las dos que más desarrollamos. Planteamos una entrevista a la rana del cuento, un teatro con marionetas, y el dibujo del mapa del reino como actividades de introducción. En la fase de comprensión se sugieren actividades de léxico y gramática sencillas, tratando, por ejemplo, los tiempos de presente y futuro. En la fase de consolidación, el profesor tiene la opción de realizar el taller del adivinador de sueños o la puesta en escena de declaraciones de amor entre animales. Finalmente, para la fase de resignificación, se cuestiona por qué el rey y la reina cambian el vestido por unos pantalones al averiguar que su hijo se casará con un chico.
\end{abstract}

Palabras clave: Homosexualidad, español como lengua extranjera, álbum ilustrado, $E l$ día de la rana roja.

\begin{abstract}
This teaching material is based on the picture book El dia de la rana roja, the story of a prince who marries another man and together adopt an orphan child. It is targeted to students of Spanish as a foreign language of any country, including the United States, with an A2 level. Picture books are useful tools to teach, not only language and grammar, but also civility and good manners in society. The proposal is divided into four phases (introduction, comprehension, consolidation, and intellectual challenge), the latter of which are explained in detail. For the introduction phase, we propose interviewing the frog in the story, having a puppet role play narrating the story, and drawing a map of the kingdom. For the comprehesion phase, we suggest grammar (present and future tense) activities and lexicon ones. For the consolidation phase, the teacher can choose between doing an activity with a mind reader or with declarations of love among animals. Finally, for the phase where their ideas are challenged, the focus is on the topic of clothing. The fact that the king and queen change the dress of the princess for a pair of trousers for the prince is challenged to show students that clothing is a mere social convention based on sex that can be questioned.
\end{abstract}

Keywords: Homosexuality, Spanish as a foreign language, picture book, El día de la rana roja. 


\section{Introducción}

En plena vorágine intercultural, el siglo XXI nos muestra una realidad social que camina, de modo inexorable, hacia la diversidad cultural, social, religiosa y lingüística. En este sentido, los contextos educativos actuales presentan la heterogeneidad como norma y no como excepción (Aguado, 2003; Domene Benito, 2015). Dicha heterogeneidad alude tanto a aspectos económicos, como culturales, de género y/o familiares. En torno a estos últimos enfocaremos nuestra atención en el presente trabajo de investigación. La familia ha sido desde la Antigüedad hasta nuestros días uno de los pilares fundamentales en la formación integral del niño como ser humano. Junto a la escuela, la familia forma un eje inquebrantable desde la etapa infantil. Sin embargo, con la llegada de la globalización, la emancipación de la mujer o la diversidad sexual una redefinición del concepto «familia» se considera esencial.

Volviendo al contexto histórico-social nos centraremos en Estados Unidos, país donde fue llevada a cabo nuestra propuesta (ver sección 4). De acuerdo con Daros (2015: 8990) si definimos la familia como una entidad compuesta por un padre trabajador, una madre ama de casa y dos hijos, los resultados que encontramos advierten claramente del cambio: solo el 7\% de las familias norteamericanas se adhieren a esta definición. Por tanto, si lo que pretendemos es mostrar la realidad social en nuestras aulas, no podemos utilizar materiales donde únicamente se ven reflejados aquellos niños que viven en estos hábitats. Este es el principal objetivo de nuestro estudio: abogar por la enseñanza/aprendizaje de español como lengua extranjera para niños donde todos los modelos y estilos familiares se traten con equidad y respeto. En esta línea argumentativa, los niños aprendientes de español como lengua extranjera (ELE), se interesan por la cuádruple relación, que consideramos indispensable en la enseñanza/aprendizaje de una lengua extranjera, aquella que se establece entre la lengua, la literatura, la cultura y la historia. Así, se produce una imbricación entre la lengua de Cervantes o Cortázar, los textos literarios expresados a través de dicha lengua y la historia y los elementos culturales que encierran dichas manifestaciones lingüísticas y literarias.

Por ende, la literatura, en este caso un álbum ilustrado, El día de la rana roja (2005), se erige como una herramienta útil y eficaz tanto para el aprendizaje de conocimientos puramente cognitivos como cívicos y emocionales a través de una historia cuya importancia estriba en el tratamiento de la familia homosexual desde una perspectiva positiva y natural.

\section{Modelos de familia}

Como ya hemos indicado en la introducción, en la era post-moderna término «familia» requiere una redefinición. Son muchos los estudiosos que enfatizan la necesidad de huir de la visión tradicional y estereotipada de la denominada familia nuclear e incluir las nuevas formas familiares (Golombok, 2000). Así las denomina Cicerchia (1999), que subraya la relevancia de adaptarse a las nuevas realidades sociales y habla de «formas familiares» donde tienen cabida tanto la familia nuclear, como la extendida, la homosexual o la monoparental. En definitiva, todo vínculo afectivo-emocional que una a seres humanos que se quieren y respetan constituye una familia. De forma similar, Daros (2015: 90-91) habla de la diversidad de «estilos familiares» en los siguientes términos: «Las naciones tecnológicamente avanzadas están actualmente llenas de una sorprendente variedad de formas familiares: matrimonios homosexuales, comunas, grupos de personas de edad que se reúnen para compartir gastos...». El United States 
Census Bureau cifra en su informe de 2015 en un 7,7\% del total el número de familias homosexuales, de las cuáles un 5,6\% están constituidas por una pareja de hombres. Ignorar esta realidad sería ignorar un porcentaje de la población que representa alrededor de 6 millones de familias.

Esta sería, a grandes rasgos, la situación ante la que nos encontramos los docentes de español como lengua extranjera al llegar al aula: distintos modelos familiares, niños distintos pero que merecen las mismas oportunidades y que se les garantice el mismo punto de partida, niños que tienen derecho a ver reflejadas sus historias familiares en los cuentos independientemente de a qué modelo pertenezcan.

\section{Enseñanza/aprendizaje de español como lengua extranjera para niños en los Estados Unidos de América: Aprendizaje significativo a través de un álbum ilustrado}

Por lo que respecta a la enseñanza/aprendizaje de español como lengua extranjera en los Estados Unidos, Heinz Kloss (1977) subraya su singularidad debido a la importancia de hechos históricos como la llegada de los primeros pobladores que colonizaron el suroeste y otras zonas que luego pasarían a formar parte del país. Sin embargo, hubo una época de animadversión hacia la lengua española y de preponderancia del inglés en estados como Nuevo México. Este hecho no favoreció ni los movimientos inmigratorios ni la implantación y desarrollo del español como lengua extranjera en las escuelas. No obstante, en la actualidad, a partir de la década de 1960 se producen avances que garantizan la supervivencia y el auge del español. Ello a pesar de que no goza de un estatus reconocido y de que la legislación norteamericana claramente favorece a la lengua inglesa. Podemos considerar que el español es una lengua viva y utilizada en Estados Unidos. En muchos casos se ha pasado de enseñarla como lengua extranjera a ser una segunda lengua para los niños (Vilar García, 2000). En consecuencia, son muchas las escuelas que integran su estudio como lengua extranjera en sus programas curriculares, tal es el caso, del estado y ciudad de Nueva York.

En estas escuelas, los niños aprenden a valorar y respetar la lengua española como un complemento básico de su formación tanto académica como personal. Ante tal esperanzadora situación, nos planteamos cómo mejorar y favorecer no solo la enseñanza de la lengua, sino también de su cultura, historia y literatura. ¿Cómo podemos unir las cuatro vertientes en una clase de ELE para niños? Uno de los soportes más eficaces es, sin duda, el álbum ilustrado. Se trata de un objeto multimodal (Kress y Van Leeuwen, 2001) que combina texto e imagen y donde ambos adquieren el mismo nivel de relevancia. Mediante este artefacto artístico y cultural, el niño aprende, indaga, descubre, piensa de forma crítica, imagina y se emociona.

Una de las investigadoras más significativas sobre álbum ilustrado en el ámbito hispánico es Teresa Colomer quien analiza el cambio de paradigma en las temáticas actuales de los álbumes: la amistad o la familia siguen siendo temas demandados y presentes en el mundo infantil y juvenil, aunque como ya hemos observado es menester huir de las visiones estereotipadas. De hecho, aún hoy en día se siguen promoviendo modelos infantiles subyugados al mundo adulto y capitalista, como es el caso de los cuentos de turismo literario de Rubbino (Kennon, 2016). Además, de acuerdo con Colomer una temática que podemos calificar como «controvertida» en la literatura infantil y juvenil ha proliferado sustancialmente: 
Se dispersan en un número infinito de temas que hace pocos años hubieran resultado insólitos como temas centrales en los libros para niños y niñas: la homosexualidad, el aborto, los maltratos y abusos sexuales, el trabajo infantil, los campos de refugiados, la anorexia, la lepra, el fútbol.

Colomer (2001 30)

La homosexualidad constituye el tema principal del álbum ilustrado en el cual se centra nuestra propuesta didáctica. Abogamos, desde aquí, por dar voz y visibilidad a los miembros del colectivo LGTB a través de la literatura como herramienta para la enseñanza/aprendizaje de ELE. Propuestas prácticas similares a la nuestra ya existen para la enseñanza de otras lenguas. Un ejemplo es la propuesta de Jódar-Sánchez (2017) para el euskera a través de la cuenta Iris eta Lila.

\section{Secuencia didáctica: El día de la rana roja}

Una vez presentado el marco teórico y la realidad social que encontramos en las aulas de español como lengua extranjera en Estados Unidos, donde la inmigración y la diversidad afectivo-sexual en torno a la familia se hace cada vez más palpable, nos disponemos a presentar el material sobre el cual hemos diseñado nuestra propuesta didáctica así como su implementación y análisis de la experiencia.

El día de la rana roja (2005) de Elexgaray relata una historia tradicional de príncipes y princesas que viven en un reino lejano pero adaptada a la actualidad. Al besar a la rana, el príncipe no encuentra una princesa, lo que encuentra es el amor verdadero. Como cualquier príncipe casadero, Héctor busca esposa. Debido a un hechizo, ese amor verdadero solo llegará cuando bese una rana roja. Tras varios intentos fallidos, la rana en cuestión llega a palacio en brazos de una niña y surge un flechazo instantáneo entre Héctor y Adrián (el príncipe en el que se convierte la rana) quienes finalmente adoptan a la niña que había hecho posible que estuvieran juntos. Los tres son el vivo reflejo de la felicidad, los tres forman una auténtica familia que no aparece unida por lazos de sangre pero sí de respeto, admiración y amor. Este es el mensaje principal de la narración: todos los modelos familiares merecen ser respetados y el amor verdadero no entiende de diferencias sexuales, culturales, religiosas o étnicas.

\subsection{Contextualización}

La presente secuencia didáctica se desarrolló durante tres sesiones con niños aprendientes de español entre 7-8 años en una escuela pública en la ciudad de Nueva York. Se trata de un grupo heterogéneo de 18 estudiantes con un nivel inicial A2.

\subsection{Estructura y motivación}

El tema de la secuencia didáctica es la familia, en general, y los modelos familiares alternativos en particular. Dentro de los comportamientos y saberes socioculturales que postula el Plan curricular del Instituto Cervantes (2007) (PCIC), equivalentes al conocimiento declarativo, se enumera el concepto y estructura de familia. Nuestra propuesta didáctica trata este tema. Más concretamente, nos centramos en desarrollar un tema que el PCIC no enumera, aunque probablemente se sobreentiende, es decir, el de las familias de padres homosexuales dentro de los tipos de unidad familiar. 
McGlashan y Sunderland (2011) analizan las estrategias que escritores de historias infantiles utilizan para presentar familias homosexuales. Son tres, la estrategia «gay», la de la «diferencia», y la del «trasfondo», de más a menos explícitas respecto a la sexualidad de los padres de los protagonistas de los cuentos. El día de la rana roja parece seguir la estrategia de la diferencia, ya que las expectativas de los habitantes del reino respecto al príncipe se reflejan en el vestido que han preparado, por ejemplo. La diferencia entre esa expectativa y la conversión de la rana en príncipe marcan la diferencia sin mencionar explícitamente que son gays u homosexuales. En nuestra propuesta combinamos las estrategias gais y de la diferencia. Niños de entre ocho y doce años ya saben de esta realidad y han escuchado palabras asociadas con la homosexualidad. Por ejemplo, entre las actividades de prelectura se plantea contrastar diferentes modelos familiares sin señalar explícitamente aquellos donde aparecen padres homosexuales, es decir, siguiendo la estrategia de la diferencia. La otra estrategia, la gay, se utiliza en la actividad de poslectura de las declaraciones de amor. El objetivo es hacer conscientes a los niños de que el amor no está limitado a parejas de distinto sexo.

Las competencias que se trabajan en esta secuencia son las siguientes:

- Habilidades lingüísticas, de léxico (amor, relaciones, imaginación) y de gramática (tiempos verbales de presente y futuro).

- Habilidades y actitudes interculturales, en concreto, habilidades como la «percepción de diferencias culturales» y el «reconocimiento de la diversidad cultural», actitudes como la empatía, la curiosidad y la apertura.

Nuestra propuesta se estructura en cuatro fases, similares a las propuestas por el Instituto Cervantes. Sin embargo, juntas forman parte del enfoque por tareas promovido en las últimas décadas (cf. Zanón, 1999) en que el plano lingüístico ha dado paso al plano comunicativo y social. Más abajo se presentan esas cuatro fases en una tabla comparativa junto con las fases que O'Malley et al. (1987) proponen desde la psicología cognitiva.

\begin{tabular}{|c|c|c|}
\hline \multicolumn{2}{|c|}{ Fases de nuestra secuencia } & Fases de O'Malley et al. (1987) \\
\hline Prelectura & Introducción & Elaboración cognitiva \\
\hline \multirow{2}{*}{ Durante la lectura } & Comprensión & Asociación \\
\cline { 2 - 3 } & Consolidación & Autonomía \\
\hline Poslectura & Resignificación & Reelaboración \\
\hline
\end{tabular}

Tabla 1. Correspondencias entre nuestras fases y las de O'Malley et al. (1987)

En primer lugar, se introduce al alumno a los conceptos básicos que se abordarán en la unidad. Se trata de la fase de introducción o presentación, que a través de actividades como las lluvias de ideas o los cuestionarios de conocimientos previos, pretende activar los esquemas de conocimiento relacionados con el tema de la familia. A continuación, el profesor ha de llevar a cabo la fase de comprensión, crucial para que el alumno sepa de qué va la clase. En la concepción de O'Malley, el alumno comienza a relacionar conceptos, tanto a nivel mental como lingüístico, en actividades aún controladas y limitadas a cierto nivel. Se establecen asociaciones entre las formas lingüísticas (palabras, sintagmas, oraciones, actos de habla, etc.) y su función. Por ejemplo, en nuestra propuesta el alumno aprende que el tiempo presente se usa para hechos y el futuro para predicciones. 
Durante la lectura del cuento no solo se pretende que el alumno entienda el contenido y algunas de las formas lingüísticas en uso, sino también que consolide esos conocimientos. Se inicia así la fase del aprendizaje autónomo que se completará más tarde con la fase de reelaboración o resignificación. En esta tercera etapa, el alumno pone en práctica plenamente sus habilidades comunicativas. El énfasis se pone sobre todo en el contenido y, ocasionalmente, en las formas. Se trata de que lo aprendido anteriormente surja de forma automática, inconsciente. Se permiten los errores. La actividad del adivinador de sueños encaja en esta fase ya que permite usar los aspectos gramaticales (tiempos verbales) y el léxico aprendidos en la fase de comprensión dentro de una situación comunicativa.

Finalmente, la fase de resignificación promueve la autonomía total del alumno al invitarle a utilizar lo anteriormente aprendido en contextos totalmente novedosos, que implican el uso de unos grados de creatividad, improvisación y confianza altos. La actividad en la que se cuestiona por qué los reyes cambian el vestido por unos pantalones sirve para romper la visión que los niños tienen de la ropa basada en el género. ¿Por qué no puede el nuevo príncipe usar el vestido que habían preparado? ¿Qué otros ejemplos existen de subversión de género en la vestimenta?

\subsection{Propuesta de actividades}

Como actividad de pre-lectura, proponemos una presentación, de tipo informal, de los diferentes modelos familiares en nuestra sociedad. Para ello el profesor puede imprimir las fotografías que adjuntamos (ver Apéndice I). Primero se les pregunta a los niños qué es una familia. Una vez contestada, se reparten las fotografías y se les pide que describan lo que ven y que si conocen a familias parecidas a las de las fotografías. Este ejercicio pone en relación lo nuevo con lo que ellos ya conocen. Se trata de una estrategia constructivista, es decir, parte de sus modelos familiares y los amplia presentando variaciones. Así mismo, se pone de relieve cual es el rasgo común en todas las fotografías, esto es, el amor, la empatía y la comprensión. Aunque la realidad no se ajuste al idealismo de estas fotografías, es importante mantener un ambiente de positividad en la clase.

A continuación, se presenta la historia de El día de la rana roja a través de sus personajes. Podemos utilizar marionetas haciendo que estas interactúen con los niños, o imágenes si no las encontramos (ver Apéndice II). Se pide a los niños que les hagan preguntas sobre cualquier cosa que quieran saber. Podemos empezar por preguntar por qué la rana es roja y no verde, o de dónde procede. Esta fase de introducción de la historia puede concluir con una actividad de dibujo. Se trata de una tarea interdisciplinar, ya que combina lengua, literatura y artes plásticas. Calculamos que todo este bloque de actividades de pre-lectura llevará entre 40 y 50 minutos.

Otra posible actividad en la parte de pre-lectura del cuento es la de dibujar un mapa del «reino diminuto que no figura en los mapas» (Elexgaray, 2005). Para orientar a los alumnos en sus descripciones, se les hará buscar en el cuento la descripción del reino, esto es, «cerca del mar, oculto entre las montañas» (ibid). Esta actividad, de tipo artístico-creativo, se puede realizar de forma individual o en grupos. Para ello, el profesor puede usar la imagen del pergamino sin escribir de la Figura 3 (ver Apéndice III). Todas estas actividades de pre-lectura ocuparán la primera sesión de la unidad didáctica. 
Dentro de las actividades del bloque de compresión durante la lectura del cuento, ya en la segunda sesión, proponemos trabajar algunos aspectos gramaticales a nivel simple. Los tiempos verbales figuran de forma recurrente en los manuales de ELE, ya que su dominio permite hablar de acciones pasadas, presentes y futuras. Con el siguiente cuadro ilustrativo pretendemos introducir los tiempos de presente y futuro ${ }^{1}$. Su uso se reforzará más adelante, en la fase de postlectura, a través de la actividad con el interpretador de sueños. Las oraciones son simples, sin subordinación ni adjetivación excesiva.

\begin{tabular}{|c|c|}
\hline Presente & Futuro \\
\hline Por la noche sueño con un papá. Me quiere & Pronto un hombre bueno te adoptará \\
\hline Sueño con un bebé. Es mi hijo & Pronto tendrás un bebé en tus brazos \\
\hline etc. & etc. \\
\hline
\end{tabular}

Tabla 2. Ejercicio con tiempos de presente y futuro

Nuestra propuesta didáctica es menos explícita respecto a las actividades de la parte desarrollada durante la lectura de El día de la rana roja dado que esta es la parte menos innovadora. Actividades de gramática o vocabulario en otros manuales servirán al profesor para esta parte.

Durante la fase de consolidación, proponemos un taller con un interpretador de sueños. El príncipe Héctor tiene sueños intrigantes al acostarse. «Cada noche, el príncipe veía repetirse el mismo sueño: se hallaba tendido en su cama y le parecía oír risas en los jardines del palacio. También oía risas entrecortadas, como cuando te hacen cosquillas. Luego una vocecita aguda gritaba “¡Papá, ven a jugar con nosotros!” y otra, como una caricia, susurraba a sus espaldas: "Deberíamos cambiar los viejos cañones por toboganes"». Esta actividad requiere más destreza lingüística y probablemente los niños a los que se dirige esta unidad didáctica no puedan realizarla en español totalmente. Lo importante es que empiecen a usar expresiones y palabras en español dentro del habla en su lengua materna. En nuestro caso, se trataba no solo de inglés, sino de otras lenguas habladas en la escuela donde la implementamos. De este modo, no asocian la lengua extranjera con la gramática sino con el ocio y el entretenimiento que proporciona una actividad como la aquí propuesta. En niveles superiores ya tendrán tiempo de formar frases enteras en español y de atender a la gramática.

Esta actividad es similar a la propuesta en Ortí Teruel y García Collado (2015). Se trata de implicar a los niños a través de una actividad teatral o de juego de roles («role playing» en inglés). Para ello, se divide a la clase en grupos de 4 alumnos. Se les explica que van a tomar la personalidad de otros. Uno de ellos será el interpretador de sueños y otro su asistente (un mago, un brujo, un duende, etc.). Los otros dos alumnos serán los clientes. Distribuyendo los roles en parejas hacemos que los alumnos se

\footnotetext{
${ }^{1}$ Dependiendo de la lengua nativa de los alumnos (el griego en Leontaridi (2001), el ruso en Mañas Navarrete (2016), el sueco en Vázquez (2015), el tailandés en Srivoranart (2011), etc.), se deberán aplicar unas estrategias u otras en la enseñanza de los tiempos de pasado y de los tiempos verbales en general. Por ejemplo, Mañas Navarrete concluye que los estudiantes rusos de ELE de nivel avanzado tienen mayor dificultad con el aspecto léxico de los verbos (por ejemplo, en los verbos explotar, correr y cocinar) más que con el aspecto gramatical (perfecto e imperfecto). Será más pertinente con ellos, pues, centrarse en el aspecto léxico. Se ha de tener en cuenta el nivel de los alumnos, ya que ciertas distinciones quizás sean demasiado avanzadas.
} 
sientan menos intimidados a la hora de hablar, ya que son pequeños y su nivel de lengua no es muy avanzado. Se propone que los niños traigan un disfraz de casa (o que se usen los del centro escolar). Si no, también se puede realizar una actividad manual en la que se creen accesorios con cartulina, tijeras, rotuladores, purpurina, papel de celofán y plastilina. A continuación, reproducimos un ejemplo de diálogo que puede servir de modelo a los niños. Como se puede observar, el lenguaje es simple ya que queremos mantenernos dentro de la «zona de desarrollo próximo» vigotskyana. En este caso, introducimos nuevas formas en español para el niño, pero sin que sean demasiado difíciles como para desmotivarlo. Vemos así mismo como se usan los tiempos de presente y futuro ya practicados en la fase de comprensión.

Príncipe: Tengo un sueño, un sueño extraño.

Interpretador de sueños: ¿Cuál es tu sueño?

Ayudante: Nosotros te ayudaremos con el significado.

Príncipe: Hay una risa, un niño o niña me llama, y luego ...

(...)

Príncipe: Por la noche pienso en voces en el castillo. Son risas, un niño o niña grita, me llama.

Amigo del príncipe: Y el tobogán, también.

Ayudante: ¿Cuál será el significado? ¿Quizás pronto tendrás familia?

Príncipe: No sé.

Interpretador de sueños: Tu mala suerte cambiará pronto ...

Planteamos una actividad alternativa a la del taller del interpretador de sueños. Se trata de una actividad de declaraciones de amor. Para ello, los niños pueden disfrazarse de animales, o crear sus propios accesorios, como en la actividad anterior. El objetivo es mostrarles un concepto de amor sin barreras, tal como se presenta el amor entre el príncipe Héctor y Adrián en El día de la rana roja. En esta actividad sí se hará mención explícita a que el amor también es posible entre personas (o animales, en este caso) del mismo sexo. Lo que pretendemos es fomentar la libertad de elección desde la infancia. Así, una vez disfrazados los niños «se ponen en la piel» de su nuevo personaje y dedican unas bonitas palabras a quien escojan. Se trata de una actividad lúdica que promueve valores como la aceptación y valoración del otro o el compañerismo.

La cuarta fase de nuestra propuesta didáctica es el proceso de re-significación. Se trata de la aplicación de los conceptos, esquemas y expresiones aprendidos durante la propuesta didáctica y los que son parte del bagaje de los niños a nuevas situaciones. Esta actividad también se sitúa dentro de la «zona de desarrollo próximo» de Vigotsky. La actividad que pone en práctica la re-significación es la siguiente. Leemos el siguiente fragmento del cuento: «[M]irando de reojo el vestido de gasa y tul que la reina Ingrid sujetaba en sus manos, señaló: "Querida, será mejor que encarguemos al sastre unos pantalones"». A continuación, ponemos a los niños en grupos de 3 ó 4 y les decimos que haremos un concurso entre ellos. Esta actividad puede llevarse a cabo en la lengua materna de los niños, ya que requiere mayor reflexión y mayor atención al contenido. Les planteamos preguntas respecto al vestido, qué tipo de prenda es, para quién, a quién va dirigido en el cuento, y en qué lo transforman los reyes. La respuesta que esperamos es que los niños digan que los vestidos son para niñas y que en el cuento los transforman en unos pantalones para que los pueda llevar el nuevo príncipe. Aquí viene la resignificación de estas ideas. Planteamos a los niños quién puede llevar pantalones, a 
lo que ellos responderán que niños y niñas (o en su defecto, hombres y mujeres). Les preguntamos entonces por qué los hombres no pueden llevar vestidos, igual que las mujeres sí que pueden llevar tanto vestidos como pantalones. Estas ideas probablemente den lugar a un intenso debate entre los niños. Esta actividad se puede complementar con ejemplos que rompen las normas sociales. Entre ellos están, por ejemplo, los hombres escoceses que llevan la típica falda escocesa («kilt») como parte de su vestimenta típica, o las faldas que a veces lleva Jaden Smith, personaje con el que seguro los niños están familiarizados (ver Apéndice III).

Como actividad complementaria, se puede repasar el vocabulario de la ropa (por ejemplo, «falda», «pantalón», «vestido», «zapatos», «ropa interior», etc.), a través de fotos en un mural. Los niños pueden crearlo ellos mismo recortando y pegando fotos junto a carteles donde aparezcan los nombres de las prendas, o el profesor puede traer uno elaborado. A partir del mural, se puede conversar con los alumnos y explicar que son las personas las que las asocian un sexo u otro a las prendas de ropa. Una actividad interesante sería retarlos a venir a clase vestidos con una prenda de ropa asociada al sexo contrario de cada uno de ellos. En general, esta actividad encaja dentro de la teoría de la performatividad en el género promovida por la feminista Judith Butler (1990). A través del lenguaje, hacemos cosas, entre ellas, «hacemos género». Las palabras y los objectos se encuentran mediados por significados y comportamientos sociales típicamente asociados con hombres o mujeres. Al utilizar las palabras que se refieren a prendas de ropa típicamente femeninas o masculinas en oraciones con pronombres del sexo contrario, por ejemplo, estamos rompiendo esa construcción social del género. Por tanto, a través de la producción de oraciones como «La falda que lleva el hombre le queda muy bien» estamos promoviendo un cambio en la percepción del niño y en la construcción social del género.

\section{Conclusiones}

En conclusión, observamos cómo la inclusión de un artefacto cultural como el álbum ilustrado desde edades tempranas favorece el desarrollo de la competencia literaria intercultural, así como la enseñanza/aprendizaje de una lengua extranjera y el refuerzo de valores morales y cívicos. Del mismo modo, mediante esta propuesta didáctica propugnamos una reivindicación de los nuevos modelos familiares y las nuevas realidades sociales basadas en la interculturalidad a través de la literatura. En concreto, dotamos al profesor de un esquema con una serie de actividades a seguir en cuatro fases diferentes (introducción, comprensión, consolidación y resignificación), con especial énfasis en las dos últimas. Todas ellas basadas en el álbum ilustrado El día de la rana roja, no solo son un material didáctico para la clase de ELE, sino también un modelo para la creación de materiales sobre otros cuentos con familias no normativas.

Se incluyen actividades de presentación del cuento, de gramática, grupales, de disfraces, y reflexivas acompañas de materiales en los anexos. En definitiva, se defiende un modelo educativo basado en valores que ayudan al desarrollo integral del ser humano como un ente que piensa, reflexiona, imagina y crea a través de fenómenos como la resignificación donde se cuestionan los significados sociales y de género de las prendas de vestir. 


\section{Bibliografía}

Aguado, T. (2003). Pedagogía intercultural. Madrid: McGraw Hill/Interamericana de España.

ButLer, J. (1990). Gender trouble. Nueva York, NY: Routledge.

CICERCHIA, R. (1999). «Alianzas, redes y estrategias: El encanto y la crisis de las formas familiares». Revista Nómadas, 11, 46-53.

Colomer, T. (2001). «La literatura infantil y juvenil actual: Entre la unicidad y la fragmentación». En E. Gil Calvo et al. (eds.), La educación lectora (pp. 37-46). Madrid: Fundación Germán Sánchez Ruipérez.

DAROS, W. (2015). «Family and education: A vision for the future from the perspective of Alvin Toffler». Tesis Psicológica, 10, N.1, 86-101.

Domene Benito, R. (2015). «The other side as a rule rather than an exception». Procedia - Social and Behavioral Sciences, 178, 66-69. DOI: 10.1016/j.sbspro.2015.03.148.

Domene BEnito, R. y JódAr SÁnChEz, J. A. (2016). El tema de la diversidad sexual en la clase de ELE para niños: El día de la rana roja. Lenguaje y Textos, 44, 83-93. DOI: 10.4995/lyt.2016.6919.

EleXgaray, C. E. (2005). El día de la rana roja. Bilbao: Editorial A Fortiori.

GolomboK, S. (2000). Parenting: What really counts?. New York, NY: Routledge.

Instituto Cervantes. (2007). Plan curricular del Instituto Cervantes: Nivel de referencia para el español. Madrid: Biblioteca Nueva.

JóDAR-SÁnCHEZ, J. A. (2017). «Pestalozzi avui: Finlàndia i l'ensenyament de l'euskera». El Guiniguada: Revista de investigaciones y experiencias en ciencias de la educación, 26, 26-33. DOI: 10.20420/ElGuiniguada.2017.134.

KENNON, P. (2016). "Childhood, power, and travel in Salvatore Rubbino's picture books: A walk in the city». Jeunesse: Young People, Texts, Cultures, 8(1), 20-41. DOI: 10.1353/jeu.2016.0001.

KLoss, H. (1977). The American bilingual tradition. Rowley, MA: Newbury House Publishers.

Kress, G. y van Leeuwen, T. (2001). Multimodal discourse: The modes and media of contemporary communication. London: Arnold.

LEONTARIDI, E. (2001). Los tiempos del pasado del indicativo en español y en griego moderno. Tesis doctoral, Universidad de Salamanca.

McGlashan, M. y Sunderland, J. (2011). «Stories featuring two-mums and two-dads families». In J. Sunderland (ed.), Language, gender, and children's fiction (pp. 142172). London: Continuum.

MaÑas NAVARRETE, Iban. (2016). La adquisición de la oposición imperfecto/indefinido por parte de estudiantes rusófonos de nivel avanzado de español LE. Tesis doctoral, Universitat de Barcelona.

O’Malley, J. M., Chamot, A. U., y Walker, C. (1987). «Some applications of cognitive theory to second language acquisition». Studies in Second Language Acquisition, 9(3), 287-306. DOI: 10.1017/S0272263100006690. 
Ortí Teruel, R., y García Collado, M. A. (2015). «Castillos en el aire». RutaELE, $10,46-56$.

SRIVORANART, P. (2011). El proceso de aprendizaje de ELE por parte de alumnos tailandeses. Condicionantes lingüisticos y culturales. Tesis doctoral, Universidad de Alcalá.

VÁzQuez, M. A. (2015). Dificultades en el uso de los tiempos del pasado imperfecto/indefinido con verbos estativos y de logro por estudiantes suecos de español como lengua extranjera con nivel A2, B1 y B2. Tesis doctoral, Universidad Antonio de Nebrija.

Vilar García, M. (2000). El español, segunda lengua en los Estados Unidos. Murcia: EDITUM.

ZANÓN, J. (coord.) (1999). La enseñanza del español mediante tareas. Madrid: Edinumen. 


\section{Apéndice I}
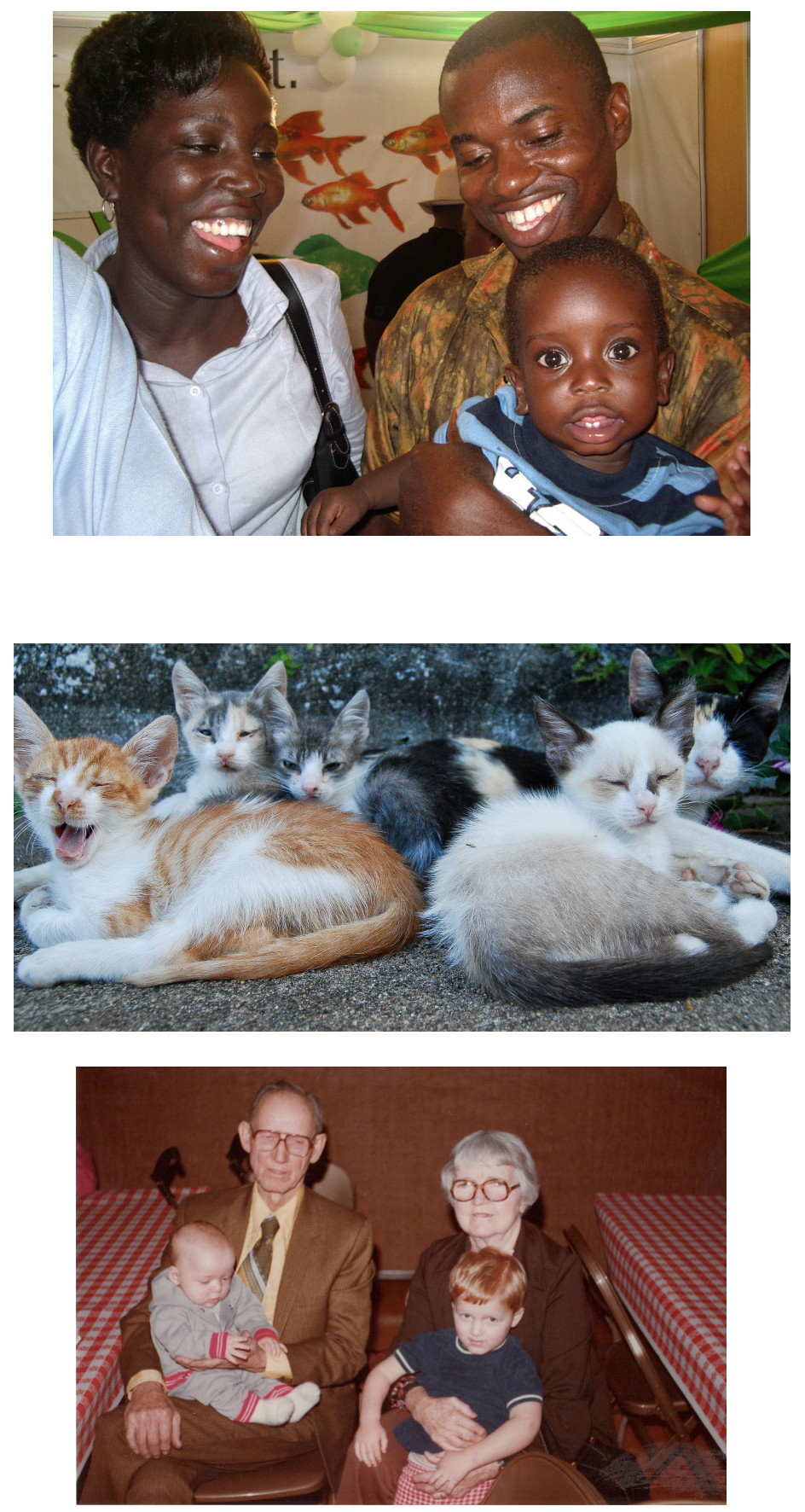

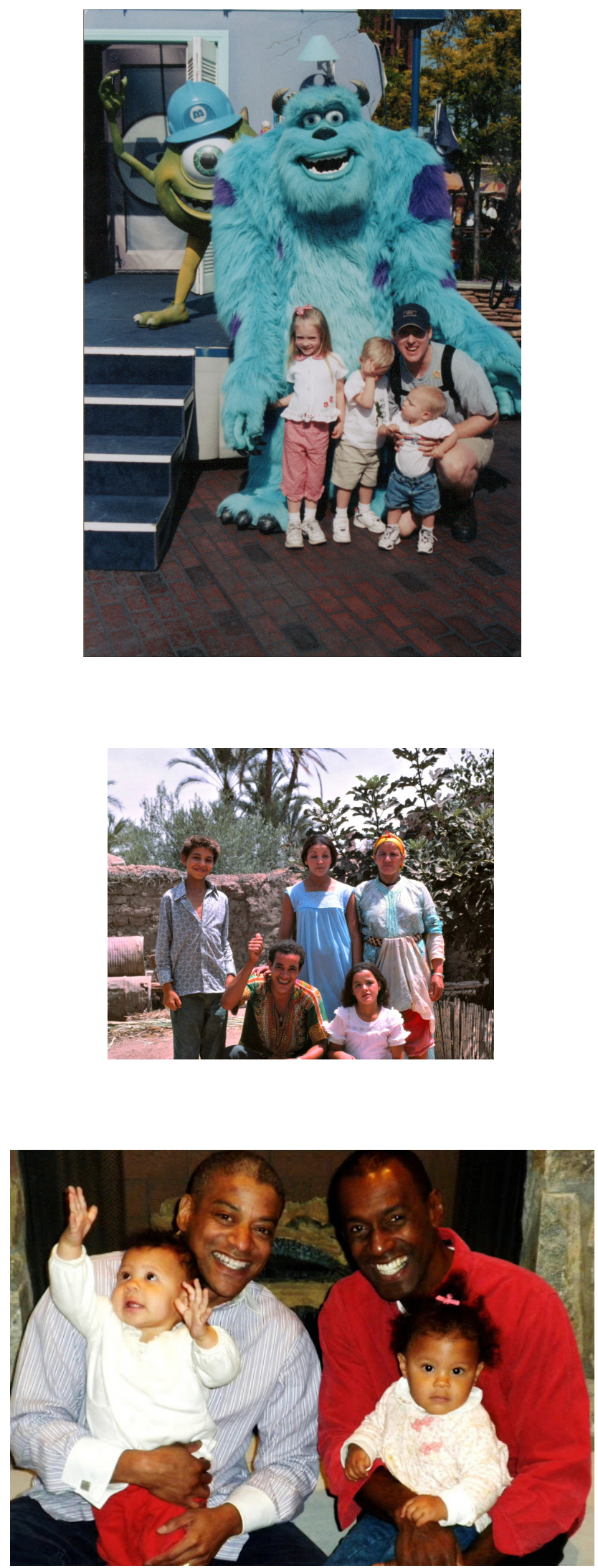


\section{Apéndice II}
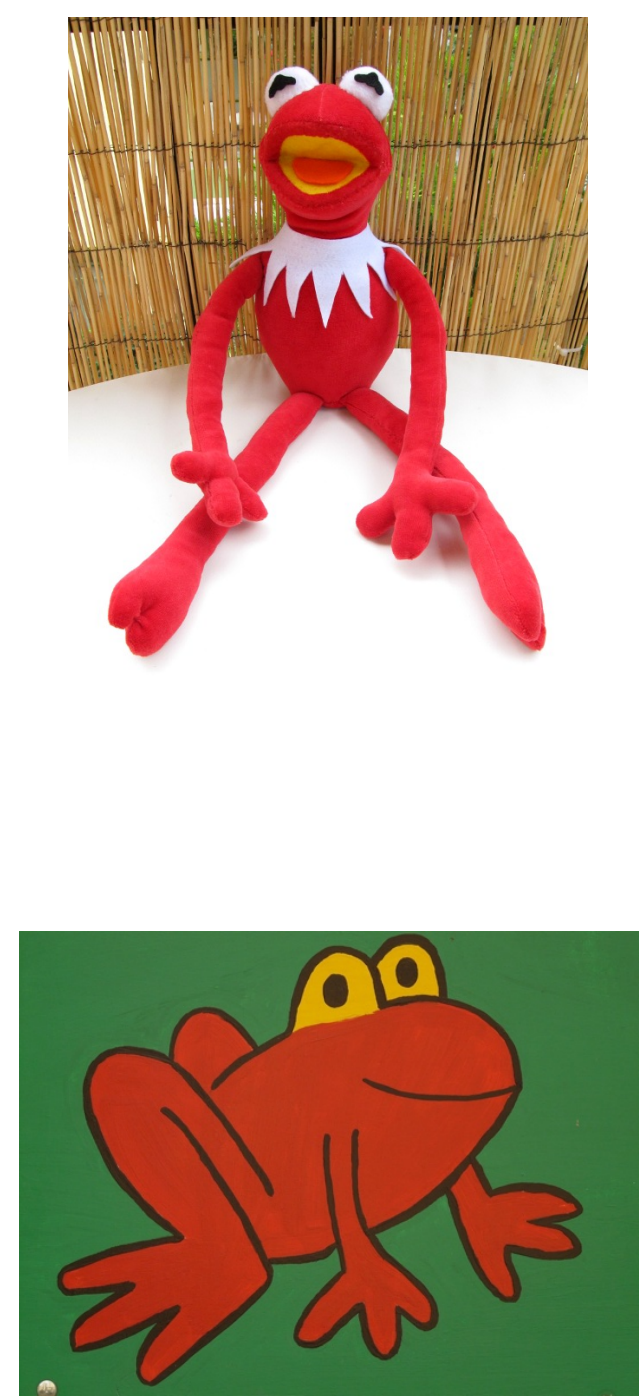

Imagen 1. Dibujo de la rana roja

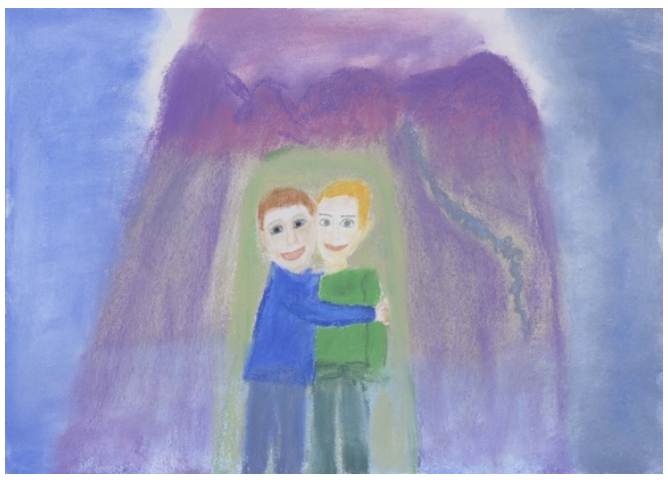

Imagen 2. Dibujo de los príncipes en su reino 


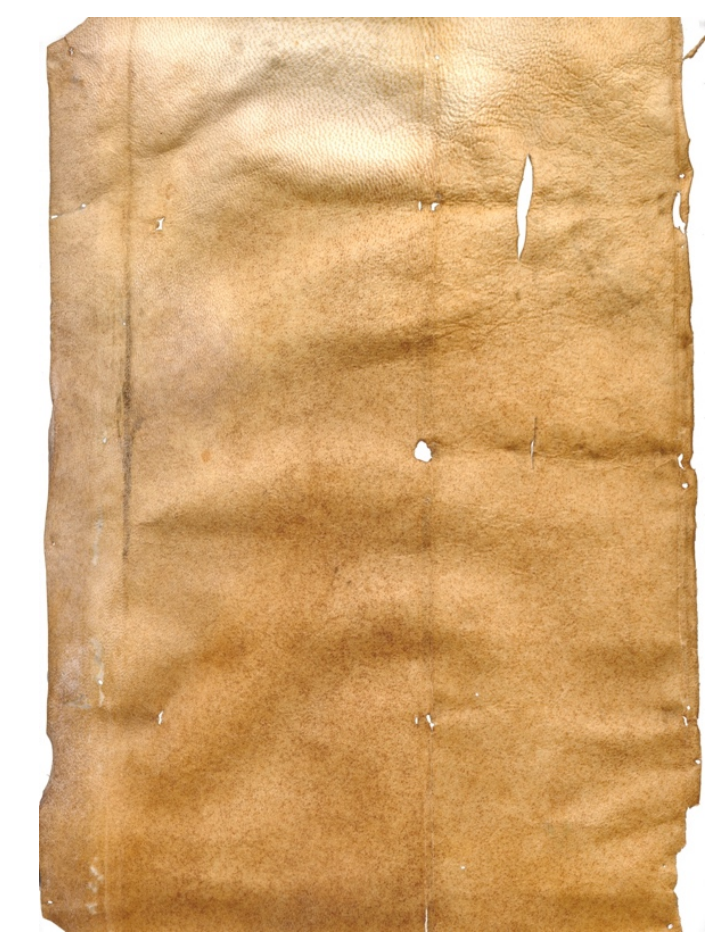

Imagen 3. Pergamino para dibujar el mapa del reino

\section{Apéndice III}
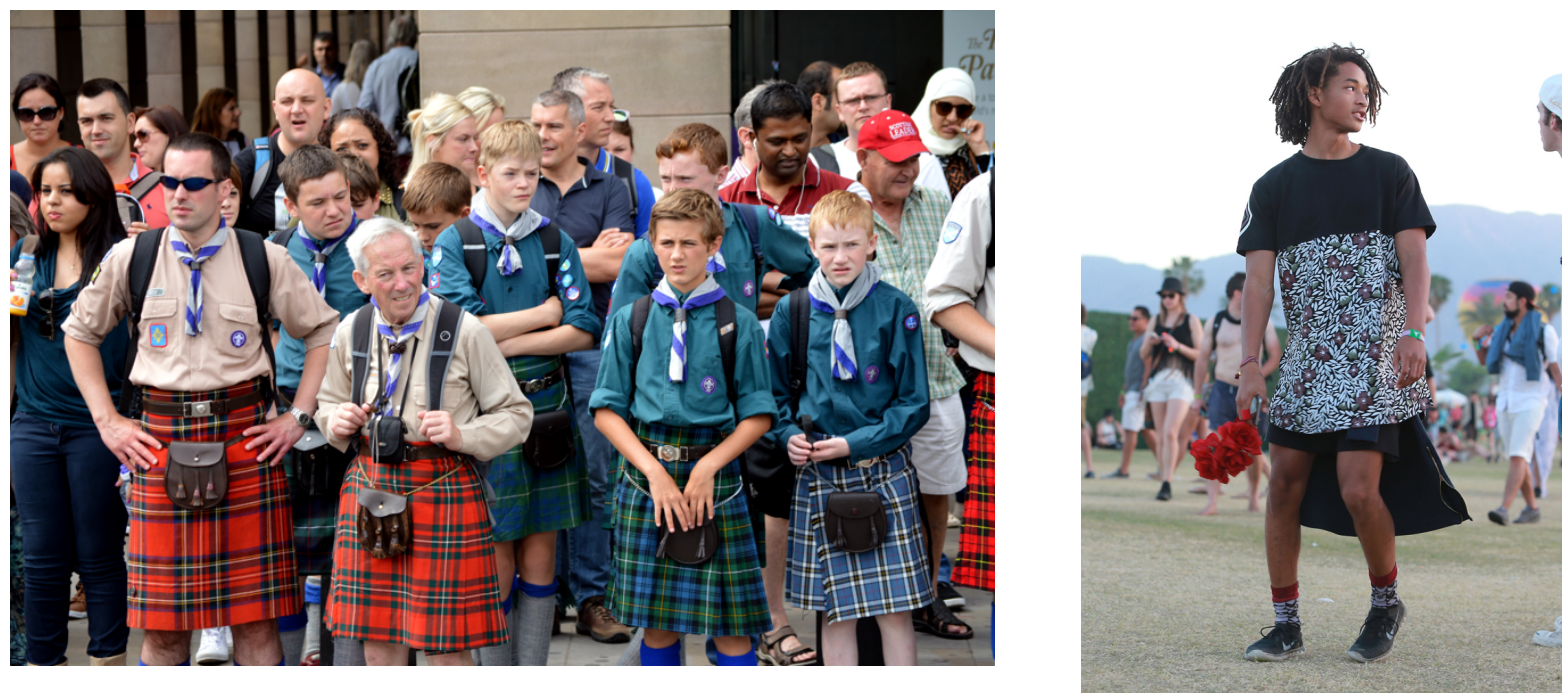